Cuestiones sociojuveniles en la encrucijada actual de los gobiernos progresistas en la región. El caso de Ecuador René Unda Lara, Nayana Román Espinel

Revista Argentina de Estudios de Juventud (N. $\left.{ }^{\circ} 11\right)$, e017, noviembre 2017

ISSN 1852-4907 | https://doi.org/10.24215/18524907e017

http://perio.unlp.edu.ar/ojs/index.php/revistadejuventud

FPyCS | Universidad Nacional de La Plata

La Plata | Buenos Aires | Argentina

\title{
SOCIOJUVENILE ISSUES
}

AT THE CURRENT

CROSSROADS

OF PROGRESSIVE

GOVERNMENTS

IN THE REGION.

THE CASE OF ECUADOR

René Unda Lara

reneunda78@gmail.com

https://orcid.org/0000-0003-4033-8762

\section{Cuestiones sociojuveniles en la encrucijada actual de los gobiernos progresistas en la región. El caso de Ecuador}

RECIBIDO 12 | 08 | 2017

ACEPTADO 31 | 10 | 2017

Palabras clave

jóvenes

desigualdades

participación política

memoria

Centro de Investigación sobre Niñez, Adolescencia y Juventud (CINA) Universidad Politécnica Salesiana

Ecuador

\section{Resumen}

La cuestión de los jóvenes, desde la perspectiva generacional, exige reflexionar sobre la memoria y problematizar, históricamente, hechos y procesos sociales. Desde esta perspectiva, los autores proponen mirar las desigualdades, su relación con algunos de los aspectos atinentes a la participación política juvenil y sus efectos sobre la democracia desde la historia reciente en el caso ecuatoriano. El análisis intenta mostrar que el enfoque y la procedimentalidad redistributivos de la política pública implementada durante la última década resultan insuficientes a la hora de construir tejido político societal si no se atienden las necesidades y las posibilidades de trabajo de memoria histórica intergeneracional enfocado en sectores poblacionales jóvenes.

\section{Abstract}

Keywords

youth

inequalities

political participation

memory
The question of young people, from a generational perspective, requires reflecting on memory and problematizing, historically, facts and social processes. From this perspective, the authors propose to look at inequalities, their relationship with some of the aspects related to youth political participation and their effects on democracy since recent history in the Ecuadorian case. The analysis tries to show that the redistributive approach and procedurality of the public policy implemented during the last decade are insufficient in the construction of societal political fabric if they are not taken into account the needs and possibilities of intergenerational historical memory work focused on young population sectors. 


\section{Cuestiones sociojuveniles en la encrucijada actual de los gobiernos progresistas en la región. El caso de Ecuador}

Por René Unda Lara y Nayana Román Espinel

Desde los estudios pioneros y referentes en torno de la cuestión de las generaciones (Mannheim, 1928; Ortega y Gasset, 1923), la preocupación por los procesos de integración y de identificaciones entre los distintos grupos de edad que conforman una sociedad en un momento o en un ciclo determinado ha sido motivo de problematización teórica sobre la que estos estudios han echado luces. Según Carmen Leccardi y Carles Feixa (2011), esta primera camada de estudios puso énfasis en el relevo generacional, lo cual es bastante comprensible en el contexto de sociedades en las que las identificaciones entre cohortes de edad estaban fuertemente vinculadas en razón de la necesidad de sucesión laboral y de transmisión de pautas valóricas sobre las que se sostenga el tejido social. En esta perspectiva, prevalece la lógica de la identidad como estructura construida por vínculos identificatorios desde un conjunto de prácticas sociales, en torno del mundo del trabajo principalmente, que configuran la noción de continuidad como categoría analítica y explicativa de procesos sociohistóricos en los que el relevo y la sucesión constituyen sus rasgos característicos. 
Tal línea de análisis resulta de enorme importancia para la comprensión de las configuraciones generacionales en términos de las condiciones y de las posibilidades de continuidad como también de ruptura. Como se ha señalado, los vínculos identificatorios que se construyen entre miembros de grupos de edad diferenciados articulan y unifican sentidos históricos en un marco cronológico determinado (Leccardi \& Feixa, 2011). Tiempo cronológico y tiempo histórico, en este sentido, producen una configuración generacional específica cuando sus agentes desarrollan prácticas compartidas y, por lo tanto, valores compartidos. La sincronía de estas dos temporalidades produce vinculación generacional que se expresa como relevo, como sucesión y como integración generacional. Tal dinámica correspondería al primer período del estudio de las generaciones enfocado en el relevo y en la coexistencia.

No obstante, y si bien el término memoria permanece prácticamente inadvertido en los estudios pioneros sobre las generaciones, la relación entre memoria e historia permite abrir posibilidades analíticas e interpretativas sobre cómo los procesos sociohistóricos que ponen en evidencia la predominancia de la continuidad como su característica central mantienen viva la memoria a través de prácticas y de sentidos compartidos entre grupos de edad distinta. Por el contrario, en las sociedades en las que los vectores dominantes de la dinámica sociopolítica se orientan hacia cambios y transformaciones, la memoria, como sustrato empírico de la historia (Aróstegui, 2007), estaría sujeta a una cierta condición de vulnerabilidad, por decirlo de alguna manera; estaría operando como un registro del presente que transcurre, más que como una cadena de acontecimientos pasados que requiere ser recuperada o invocada en función de instalar en el sentido común la conciencia de que un estado de cosas se ha modificado. $\mathrm{Y}$ esto porque los hechos presentes, habiendo sido producidos gracias a la acción de determinadas fuerzas sociales que incidieron en el pasado reciente para transformarlo, no estarían siendo asimilados ni percibidos como hechos que representan una ruptura en un orden de cosas, sobre todo por parte de segmentos mayoritarios de las jóvenes generaciones que son quienes, por ejemplo, han experimentado de modo directo los beneficios de las políticas redistributivas impulsadas por los gobiernos progresistas de la región.

A la luz de los escenarios sociopolíticos generados en América Latina en el contexto de los gobiernos progresistas, parece que la configuración de una conciencia generacional capaz de establecer puntos de inflexión o cortes temporales que delimiten con cierta claridad los ciclos económicos y políticos, ocurridos y en curso, enfrenta en cada uno de estos países serios problemas, si se atiende, por ejemplo, a resultados 
de procesos democráticos tales como las elecciones presidenciales de noviembre de 2015 en la Argentina; el referéndum boliviano sobre la posibilidad de reelección presidencial, en febrero de 2016; la apretadísima ventaja en la votación de las recientes elecciones presidenciales en Ecuador, en abril de 2017; e, incluso, la muy cuestionada destitución vía Senado de la expresidenta de Brasil, Dilma Rousseff, en abril de 2016.

A partir de reconocer que la consolidación y la radicalización de las políticas redistributivas implantadas en estos países es un proceso que entraña una enorme complejidad por todo lo que supone e implica, se reconoce, también, que uno de los factores más relevantes en esta dinámica se relaciona con cierta fragilidad y con ciertas dificultades en la transmisión de ese particular tipo de memoria histórico-política, de modo tal que permita reconocer y valorar el antes y el después de un determinado ciclo político. Y, con ello, contribuir al fortalecimiento de un tejido ciudadano que, teniendo conciencia plena de sus derechos a partir de su ejercicio, pueda ser partícipe de su ampliación y de su profundización.

Sin duda, uno de los déficit en la constitución de un tejido social y político que no solo viabilice la posibilidad de que segmentos mayoritarios de la sociedad ofrezcan condiciones para el sostenimiento de los procesos que llevan a cabo los regímenes progresistas, sino que genere cambios significativos en la ecuación Estado-sociedad, radica en la debilidad de aquello que desde distintos lugares se ha nombrado como memoria histórica. En tal desafío es que las relaciones intergeneracionales juegan un papel central en tanto interacciones que, reconociendo el diferencial cultural de las distintas cohortes generacionales, es decir, aquello que pueda suponer rupturas, sean capaces de promover vínculos identificatorios que planteen líneas de continuidad en aquello que signifique el afinamiento de un proyecto político cuyo horizonte de realización esté basado en la igualdad y en las condiciones de vida dignas para todos los miembros de la sociedad.

Sin embargo, intentar elaborar un análisis que articule memoria y desigualdades desde una perspectiva generacional supone, como se ha señalado, considerar las múltiples aristas e implicaciones que cada uno de estos aspectos comporta; en particular para los temas de participación y de democracia. Podría decirse que la activación de la memoria histórica (cronológica y de sentido) resulta muy difícil de pensar al margen de procesos participativos centrados, principalmente, en las dinámicas territoriales y de politización (Vommaro, 2015), en tanto agregación de demandas que se generen desde 
sectores sociales que hayan experimentado los beneficios de políticas diseñadas para combatir las desigualdades y las brechas de inequidad que en América Latina son las más pronunciadas de todo el planeta (Oxfam, 2017).

Por lo anterior, en esta aproximación analítica se privilegia el abordaje de los cambios y la evolución en la disminución de las desigualdades a partir de 2007 en el caso ecuatoriano, año en el que el gobierno presidido por Rafael Correa asumió la conducción gubernamental del Estado.

\section{La relación de las desigualdades con la participación política juvenil}

La constatación del incremento de la desigualdad como fenómeno global, a contrapelo de las declaraciones de distinto tipo y alcance que se han hecho desde numerosos espacios institucionales nacionales y supranacionales acerca de la lucha contra la pobreza, no ofrece mayores dificultades. Los datos y las informaciones producidos por varios organismos, entre ellos Oxfam, ponen en evidencia la cada vez más intensificada concentración de la riqueza. La «economía del 1\%», que según el informe de Oxfam 2017 no solo pone en riesgo la existencia digna del 99\% restante de la población mundial, sino la posibilidad misma de sostenibilidad planetaria en términos ecológicos.

Para fines de nuestro análisis, aludiremos únicamente a dos de los aspectos cuestionados en el mencionado informe y que tienen relación directa con algunas de las políticas implantadas desde 2007 por el gobierno de la Revolución Ciudadana. El primero de ellos es la profunda reforma del sistema de rentas internas a través de las modificaciones sustantivas de los criterios, la ponderación y la procedimentalidad de la recaudación tributaria bajo una perspectiva de corte socialdemócrata, ensayada y probada ya en otros contextos, según la cual quien más rentas obtiene más contribuye al fisco. Tal criterio, que posibilita la concreción de políticas redistributivas en beneficio de los sectores históricamente empobrecidos (quintiles 1 y 2, caso Ecuador), pero no solo de estos, y que no representaría novedad alguna en los países en los que el Estado ha venido desempeñando un rol regulador de la actividad económica en general, supuso, en el caso ecuatoriano, la apertura y la multiplicación de escenarios de confrontación entre la recién instalada estructura estatal y diversos sectores de la sociedad que tenían a las tradicionales oligarquías como uno de sus actores principales. 
Como es conocido, un modelo de recaudación tributaria que privilegia los denominados impuestos directos $-\mathrm{y}$, específicamente, el impuesto a la renta- funciona como elemento de contención frente a la inevitable concentración de la riqueza y, simultáneamente, como condición de posibilidad para la ejecución de su misma redistribución en favor de grupos y de sectores postergados y en situaciones de vulnerabilidad; entre ellos, los/as jóvenes de entre quince y veinticuatro años a quienes los problemas de desempleo, por ejemplo, afectan en doble y en triple proporción según índices promedio (MIES, 2012). Esto no solo significa contención de la acumulación concentradora de riqueza, sino también generación de estructuras de oportunidad para sectores pauperizados que redunda en disminución de las desigualdades y de las brechas de inequidad.

El segundo de los aspectos que retomamos en este trabajo, como uno de los que mayor conflictividad produjo, es el referido a la gratuidad de la educación y de la salud públicas. En un país en el que la dinámica de privatización de los servicios públicos fue impulsada entusiastamente por el Estado neoliberal, muy en especial durante los tres lustros anteriores al gobierno de Rafael Correa, la implantación de este conjunto de políticas debió pasar, entre otras dificultades, por un proceso inédito, como fue el Proceso Constituyente, que finalmente fue aprobado el 28 de septiembre de 2008. Como muestran los datos oficiales, la inversión pública en educación y en salud se incrementó hasta en catorce y quince veces más entre 2007-2013 en relación con todos los gobiernos anteriores desde el retorno a la democracia, en 1979 (Unda Lara \& Llanos, 2013)

Los doce procesos electorales y plebiscitarios que se han realizado desde 2006, y en los que se ha ratificado la presencia de Alianza PAIS como primera fuerza política del Ecuador, han representado de manera inobjetable el apoyo de los sectores mayoritarios del país. Sin embargo, a la fatiga que normalmente caracteriza a un gobierno que ha permanecido diez años en el ejercicio de gobierno y que lo hará cuatro años más con el presidente electo Lenín Moreno, debe sumarse la inexistencia de elementos que evidencien que en las nuevas generaciones, en particular en aquellas que ingresaron al sistema de educación pública en 2007 , se haya instalado una suerte de memoria del pasado reciente que permita diferenciar con suficiente claridad el Ecuador previo y el posterior a 2007. Desde esta perspectiva, se abren nuevos interrogantes y posibilidades analíticas acerca de las desigualdades generacionales en términos informativos. 


\section{La producción de desigualdades bajo la forma pobreza}

Una de las expresiones tipo de la desigualdad es la pobreza producida por deficitarias o por inexistentes políticas de redistribución de la riqueza. Una política pública orientada hacia el mejoramiento de las condiciones de vida del conjunto de la sociedad debe enfocarse en reducir las brechas de desigualdad a través de una serie de mecanismos articulados que regulen las tendencias concentradoras de acumulación de la renta y que generen estructuras de oportunidades y de accesos a bienes y a servicios públicos como condición fundamental de un grado de cohesión social que permita el desarrollo pleno de sus miembros.

Evidentemente, esta perspectiva de cuño republicano encuentra severos obstáculos en el marco general de contextos institucionales, frente a las posiciones de distintas organizaciones políticas y sociales, que se ubican no solo en la derecha del espectro político ideológico, así como en las mismas prácticas políticas de una diversidad de actores, con lo cual el proceso general de producción de políticas públicas de carácter redistributivo adquiere altos niveles de complejidad. No obstante, la consigna de la «lucha contra la pobreza» - visibilizada globalmente a partir del Consenso de Washington, en 1989-, instaló un potente y un extendido sentido común, tanto en las lógicas de gubernamentalidad como en las dinámicas de intervención de distintos actores, que incluyeron la cooperación internacional, organizaciones no gubernamentales, instancias paraestatales, e incluso asociaciones de carácter filantrópico, y que constituyeron un verdadero dispositivo, en el sentido foucaultiano del término.

En América Latina, y específicamente en Ecuador, las prácticas y los discursos heterogéneos de tal dispositivo casi nunca se interrogaron, sin embargo, por intervenciones sobre el modelo de acumulación concentradora de la riqueza, asunto que habría significado abrir paso a la posibilidad de pensar en el tema redistributivo. No es sino con el advenimiento del gobierno de la Revolución Ciudadana, en 2007, que la impronta redistributiva se diseñó y se planificó en relación directa con el control de la concentración de la riqueza, y se ejecutó por vía de la reforma tributaria, cuestión bastante distinta a la redistribución aplicada en el primer lustro de la década de 1970 durante la primera fase del «boom» petrolero administrado por el gobierno nacionalista y revolucionario del Gral. Rodríguez Lara, en el que la redistribución operó a través de mecanismos directos en el marco general de una economía boyante y sin modificaciones al sistema de tributación. 
La llegada del gobierno de la Revolución Ciudadana significó la puesta en acto de mecanismos redistributivos orientados, principalmente, a la cobertura y al acceso de sectores populares a servicios públicos que permitan el ejercicio efectivo de los derechos de educación y de salud. Esto supuso, a su vez, reformas amplias y problemáticas que pudieran ser viabilizadas con el propósito de reducir la pobreza.

Desde este marco general, en lo que sigue se propone un acotado análisis de datos estadísticos sobre pobreza en el país, en particular de los índices de pobreza por consumo. Se identifica una tendencia común, antes de 2006, y una opuesta, después de 2006; un cambio que se debe, principalmente, a la implementación de políticas públicas tales como una clara política fiscal, mejoras en los salarios de la población, disminución de la tasa de desempleo y ampliación significativa de afiliados a la seguridad social. Medidas como estas no solo reducen los indicadores de pobreza, sino también la brecha de desigualdad, aunque, inobjetablemente, las condiciones de la población joven comprendida entre los 15 y los 24 años acusan mayores niveles de desigualdad que otros grupos de la población, más allá de que su situación relativa sea mejor que en décadas anteriores.

En el GRÁFICO 1 se muestra un balance histórico de las condiciones de pobreza por ingreso en el país desde 1988 hasta 2005. En el eje izquierdo se detalla el índice de pobreza y en el derecho el índice de inflación. En su lectura debe considerarse que en 2000, en medio de la crisis económica más profunda en la historia republicana del Ecuador, el gobierno presidido por Jamil Mahuad adoptó el esquema económico monetario de dolarización. Por ello, el gráfico muestra un descenso abrupto y acelerado en relación con la inflación (Albornoz, 2011). 
GRÁFICO 1 | Pobreza e inflación (1988-2005)

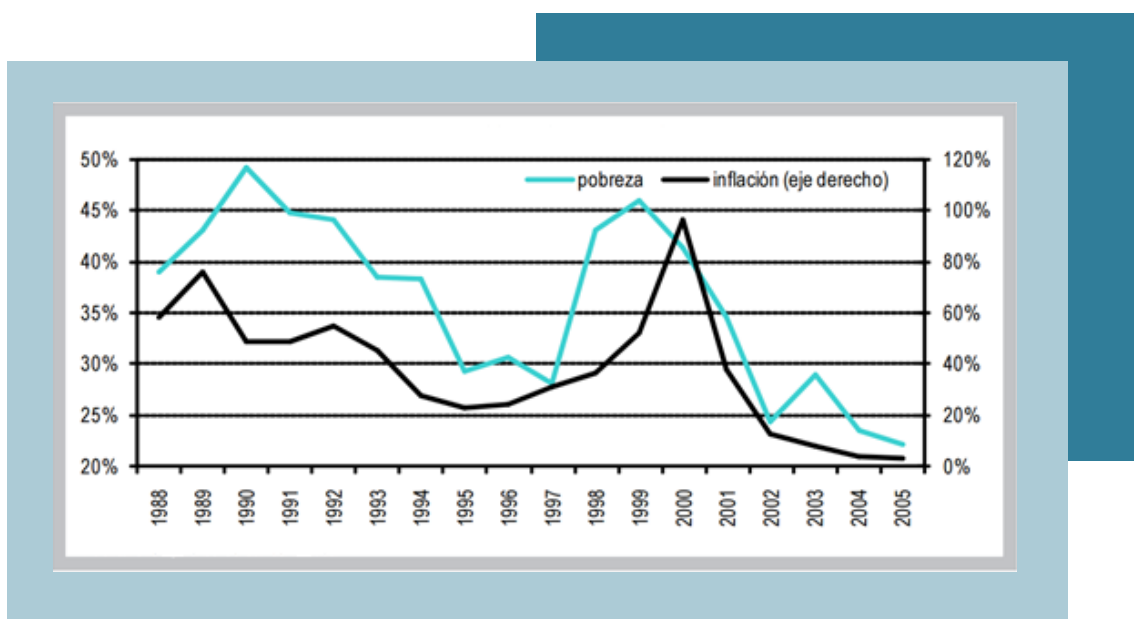

Fuente: Sistema Integrado de Indicadores Sociales del Ecuador (SIISE) y Banco Central del Ecuador

En el CUADRO 1 se presentan los datos de los años comprendidos entre 1995 y 2014. Pueden distinguirse dos períodos: el primero, de 1995 a 2006, con una tendencia ascendente tanto de los niveles de pobreza como de los índices de desigualdad; el segundo, de 2006 a 2014, con un descenso en los índices de pobreza y de desigualdad.

CUADRO 1 | Pobreza por consumo y desigualdad de consumo (1995-2014)

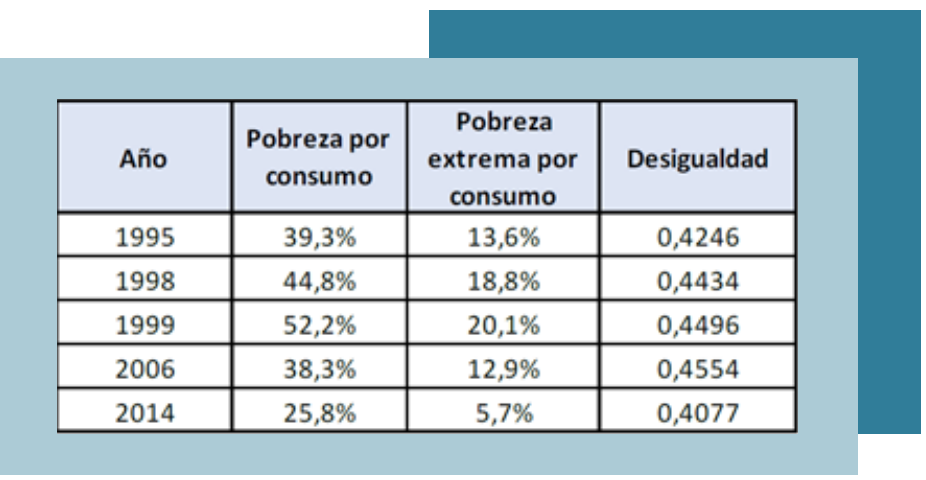

Fuente: Instituto Nacional de Estadísticas y Censos (2014) 
En el siguiente reporte del Instituto Nacional de Estadísticas y Censos (INEC, 2014), se muestra el índice de desigualdad, medido a través del Coeficiente de Gini, en los períodos 1995-1998, 1999-2006 y 2007-2014. En los dos primeros el índice de desigualdad subió, y a partir del tercer período el índice de Gini disminuyó. Esto se debe, principalmente, a la aplicación de políticas públicas redistributivas (aumento de salarios, seguridad social, política fiscal). En el período de 1999-2006 se observa una disminución de los niveles de pobreza, mas no del índice de Gini, lo cual significa que las desigualdades permanecen inalteradas.

\section{GRÁFICO 2 | Variación de la desigualdad por consumo (1995-2014)}

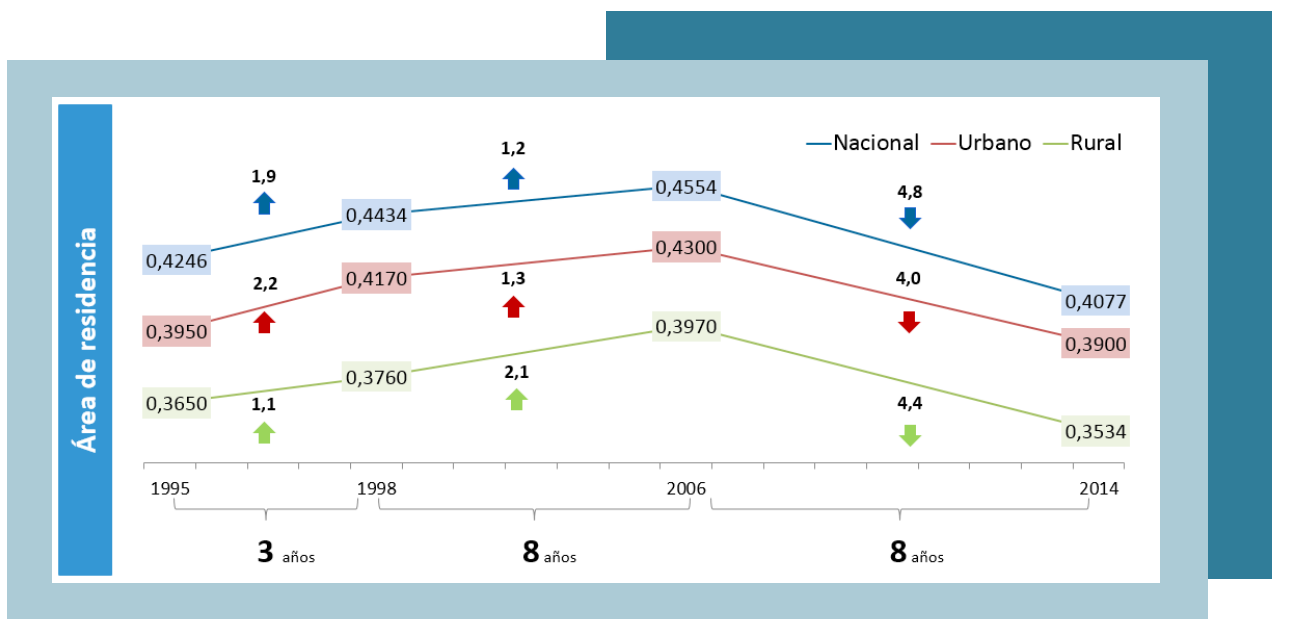

Fuente: Instituto Nacional de Estadísticas y Censos (2014)

En el período 1995-2006, los índices de pobreza y de extrema pobreza no solo no se redujeron, sino que se mantuvieron con una tendencia ascendente. Se trata del período en el que el gobierno de Sixto Durán Ballén y de Alberto Dahik entró en su fase final, y se consolidó la implantación de los ejes dinamizadores del paquete de políticas neoliberales: desregulación del sistema financiero, privatización y desmontaje institucional de lo público, y flexibilización laboral. En esta década se sucedieron siete presidentes y se produjeron dos derrocamientos presidenciales (Bucaram, 2007, y Mahuad, 2000, acusado de peculado). 
Como se observa en el GRÁfICo 3, en el período 2006-2014 los niveles de pobreza por consumo se redujeron significativamente. Para 2014, la pobreza extrema se redujo a la mitad; menos dramático, pero igual de importante, fue la reducción en los índices de pobreza, que pasaron de 38,3\% en 2006 a 25,8\% en 2014 (INEC, 2016).

\section{GRÁFICO 3 | Tendencias de pobreza en base al consumo (1995-2014)}

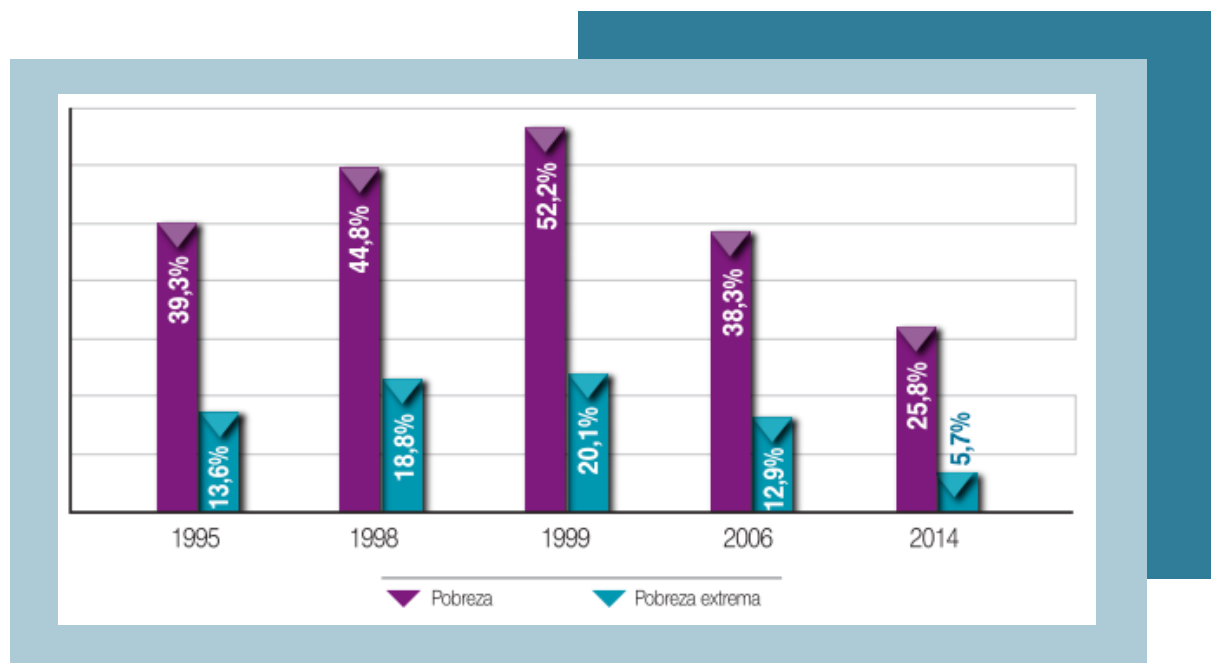

Fuente: cálculos de los autores usando ECV 1995-2014

El CUADRO 2 indica la profundidad y la severidad de la pobreza en el Ecuador y la evolución de este índice por población (urbana / rural, regional, nacional), mediante una comparación entre el año 2006 y el 2014. En las filas centrales se puede observar, además, la brecha que existe entre estos dos períodos. 
CUADRO 2 | Incidencia, profundidad y severidad de la pobreza en Ecuador (1995-2006)

\begin{tabular}{|c|c|c|c|c|c|c|}
\hline & \multicolumn{2}{|c|}{ Incidencia (\%) } & \multicolumn{2}{|c|}{ Brecha (\%) } & \multicolumn{2}{|c|}{ Severidad (\%) } \\
\hline & 2006 & 2014 & 2006 & 2014 & 2006 & 2014 \\
\hline \multicolumn{7}{|l|}{ Pobreza } \\
\hline Urbana & 24,9 & 15,5 & 6,8 & 3,6 & 2,6 & 1,2 \\
\hline Rural & 61,5 & 47,3 & 24,5 & 15,1 & 12,9 & 6,7 \\
\hline Sierra & 33,8 & 24,5 & 12,0 & 7,0 & 5,9 & 2,9 \\
\hline Costa & 40,3 & 24,8 & 12,6 & 6,4 & 5,3 & 2,4 \\
\hline Amazonia & 59,7 & 47,7 & 32,3 & 19,2 & 21,4 & 10,2 \\
\hline Nacional & 38,3 & 25,8 & 13,3 & 7,3 & 6,4 & 3,0 \\
\hline \multicolumn{7}{|c|}{ Pobreza extrema } \\
\hline Urbana & 4,8 & 1,9 & 0,9 & 0,3 & 0,3 & 0,1 \\
\hline Rural & 26,9 & 13,8 & 8,4 & 3,3 & 3,8 & 1,2 \\
\hline Sierra & 12,2 & 5,6 & 3,5 & 1,2 & 1,5 & 0,4 \\
\hline Costa & 10,8 & 4,2 & 2,3 & 0,8 & 0,7 & 0,2 \\
\hline Amazonia & 39,6 & 21,8 & 18,5 & 6,8 & 10,6 & 2,9 \\
\hline Nacional & 12,9 & 5,7 & 3,6 & 1,3 & 1,6 & 0,4 \\
\hline
\end{tabular}

Fuente: cálculos de los autores usando ECV 2006-2014

Los datos de pobreza en la población ecuatoriana tuvieron una transformación significativa a partir de 2007, cuando comenzaron a implementarse las políticas propuestas en el Plan Nacional del Buen Vivir.

A continuación, se revisan datos de pobreza por consumo según el registro histórico de datos con el fin de apreciar su evolución y de introducir en el análisis un factor determinante, a saber: la importancia de las remesas enviadas por quienes tuvieron que salir del país entre 1999 y 2001 a causa de la crisis detonada por el feriado y el salvataje bancario, y por la dolarización. Como muestra el GRÁfICO 4, entre 1998 y 2006 la pobreza por consumo descendió en un promedio de aproximadamente el $14 \%$ por efecto del envío de remesas, segunda fuente principal de ingresos del país en aquel período (INEC, 2014), cuestión que para nada obedece a la implantación de políticas públicas con enfoque redistributivo. Desde la perspectiva de las desigualdades intergeneracionales, varios estudios muestran los perversos efectos que la ola migratoria provocó en la niñez y juventud cuyos progenitores se vieron obligados a buscar fuentes de ingresos económicos en países como España e Italia (Gaitán, 2010). 
El indicador de pobreza por consumo permite entender cómo han variado los índices de pobreza, pero no necesariamente indica la existencia y la dirección de medidas de redistribución de la riqueza. A partir de 2007, la pobreza por consumo descendió a un promedio de aproximadamente $33 \%$ por la confluencia de una serie de factores favorables y, en buena medida, estructuralmente consistentes (creciente inversión pública en salud, en educación y en infraestructura vial e hidroeléctrica, ampliación de la cobertura y la afiliación a la seguridad social, ampliación de condiciones de empleabilidad y acceso a nuevas fuentes de crédito para unidades productivas medianas y pequeñas), hasta otros de carácter contingente y con menor posibilidad de control (como el alto precio de los commodities, que se extendió hasta 2012).

Según datos del INEC, en el período de 1995 a 1998 la pobreza se incrementó en un 13,9\%, mientras que en el de 1998 a 2006 disminuyó en un 14,5\%. Este es el período de la crisis financiera de 1999 y lo que sigue es el proceso de recuperación, por lo que se observan los datos favorables correspondientes. A partir de 2006 y hasta 2014 la pobreza se redujo en un $32,6 \%$. En el mismo informe se señala que el éxito se debe a políticas públicas de redistribución. Ejemplo de estas políticas es el aumento en los salarios de la población, los mismos que constituyen en la actualidad el 70\% del ingreso de los hogares (INEC, 2014).

GRÁFICO 4 | Variación de la pobreza por consumo (1995-2014)

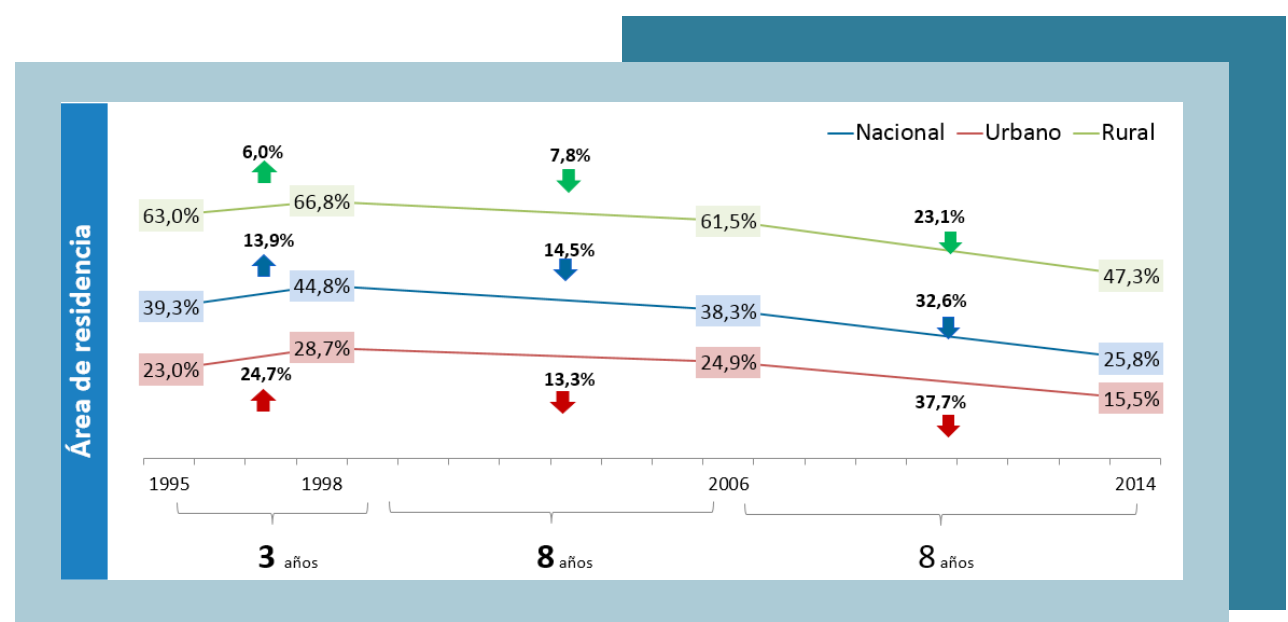

Fuente: Instituto Nacional de Estadísticas y Censos (2014) 
En el informe de pobreza del INEC (2014) se observa que los índices de extrema pobreza por consumo bajan significativamente en el período 2006-2014, disminuyendo en un 55,4\%, y que se incrementan en los períodos de 1995 a 1998, aumentando en un 38,2\%; se puede observar disminución de este índice del 31,6\% en el período de 1998 a 2006.

GRÁFICO 5 | Pobreza extrema por consumo (1995-2014)

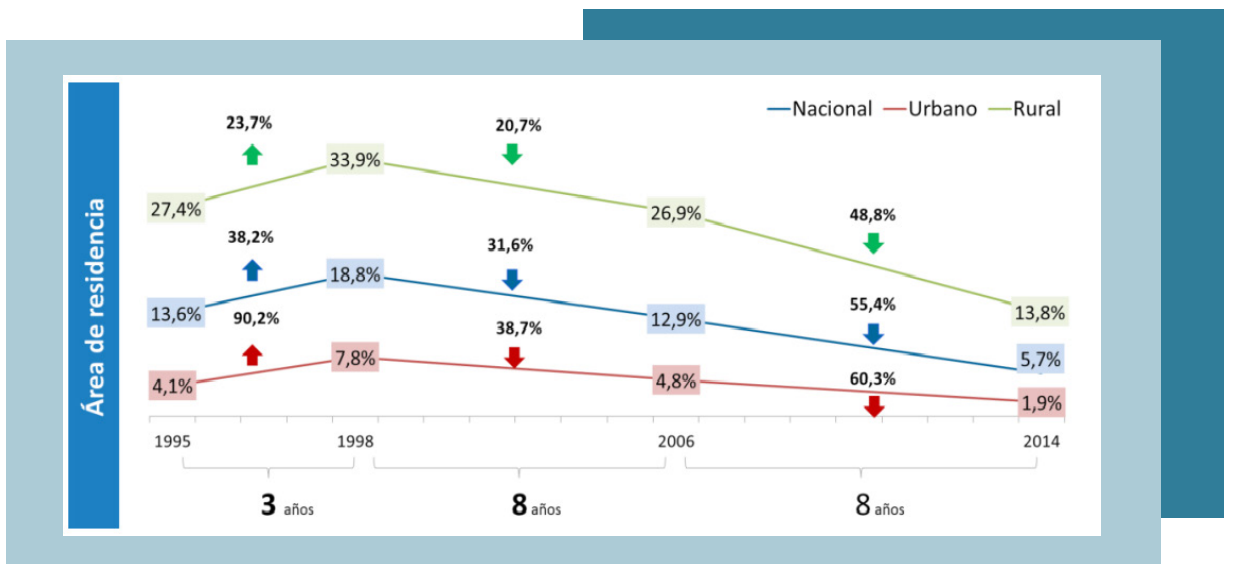

Fuente: Instituto Nacional de Estadísticas y Censos (2014)

\section{La participación política como participación de la riqueza}

Uno de los problemas derivados de la estructura de desigualdad es la concentración tendencial de los recursos en ciertos sectores de la población. Como se afirma en el «Informe sobre Jóvenes Ecuatorianos en Cifras» (MIES, 2012):

La distribución intergeneracional de recursos sigue beneficiando ampliamente a la población adulta y, en cierta medida, también a la población adulta mayor. [...] se trata, sin duda, de un problema estructural, que se constata en la mayor parte de los países latinoamericanos (p. 2). 
Para el Ecuador, tal constatación representa un problema central, en razón de la actual composición demográfica del país, ya que por primera vez en su vida republicana los y las jóvenes son mayoría. Similar situación se observa respecto de la inversión pública dirigida a infancia y a jóvenes, en la cual este último segmento poblacional aparece en franca desventaja desde una mirada comparativa, aunque evidentemente pueden esgrimirse una serie de argumentos válidos en una y otra dirección. Pero quizás lo más llamativo dentro de la estructura de desigualdad es que los datos muestran concentración tendencial entre los mismos jóvenes, en perjuicio de las mujeres, situación que se agrava cuando se trata de mujeres jóvenes indígenas o afrodescendientes.

Al respecto, cabe anotar que el necesario debate acerca de si las políticas sectoriales son suficientes para lograr el bienestar social de la población joven, o si es necesario evaluar con mayores elementos la pertinencia de elaborar políticas específicas de juventud, es prácticamente inexistente; incluso en el momento actual, en el que el presidente electo, Lenín Moreno, ha anunciado en su programa de gobierno la creación de la Secretaría de la Juventud como instancia rectora de las políticas públicas de juventud en Ecuador.

Como dato adicional disponible, esta vez correspondiente a pobreza por condición de Necesidades Básicas Insatisfechas (NBI), se observa que en promedio la población joven es el 30,4\% de la población en condición de pobreza y el 22,3\% de la población en condición de extrema pobreza, mientras que el 47,3\% de la población no es pobre (INEC, 2010).

\section{CUADRO 3 | Condición de pobreza por Necesidades Básicas Insatisfechas (NBI) según grupos de edad y de sexo (2010)}

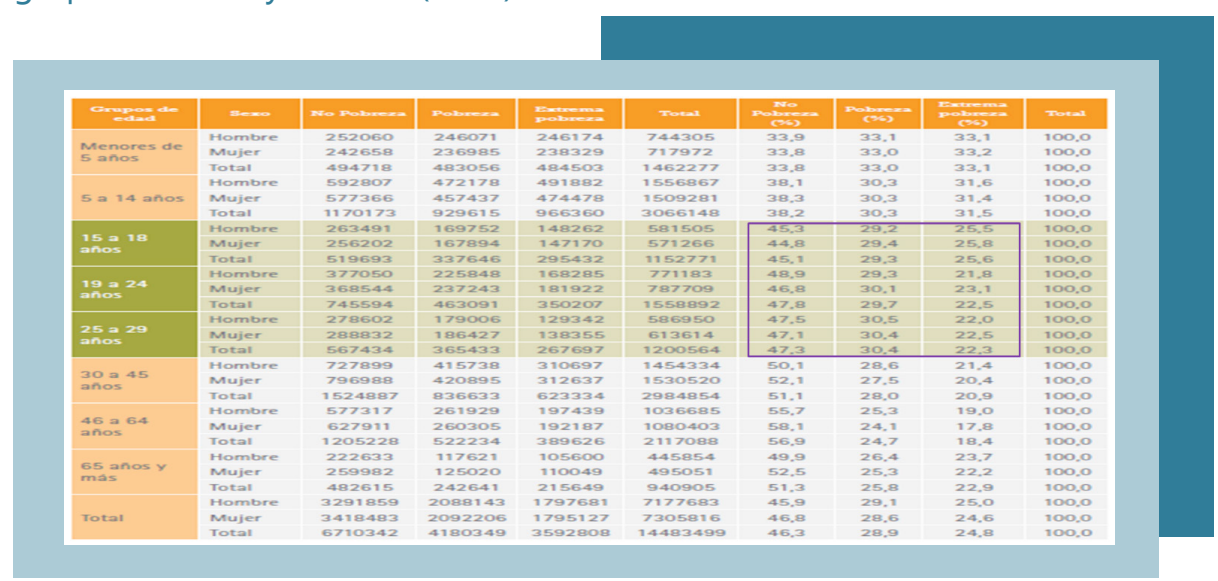

Fuente: Censo de Población y Vivienda 2010, INEC 
El GRÁfICO 6 muestra las condiciones de la población joven por NBI, según rango de edades: el 29,3\% corresponde a la población de 15 a 18 años; el 29,7\%, a la población de 19 a 24 años; y el 30,4\%, a la población de 25 a 29 años. En el caso de la población que se encuentra en extrema pobreza, las edades más jóvenes tienen el porcentaje más alto, con el 25,6\%; las personas que se encuentran entre los 19 y los 24 años, el 22,5\%; y la población de entre 25 y 29 años un porcentaje de 22,3\% (INEC, 2016).

\section{GRÁFICO 6 | Jóvenes y pobreza por Necesidades Básicas Insatisfechas (NBI)}

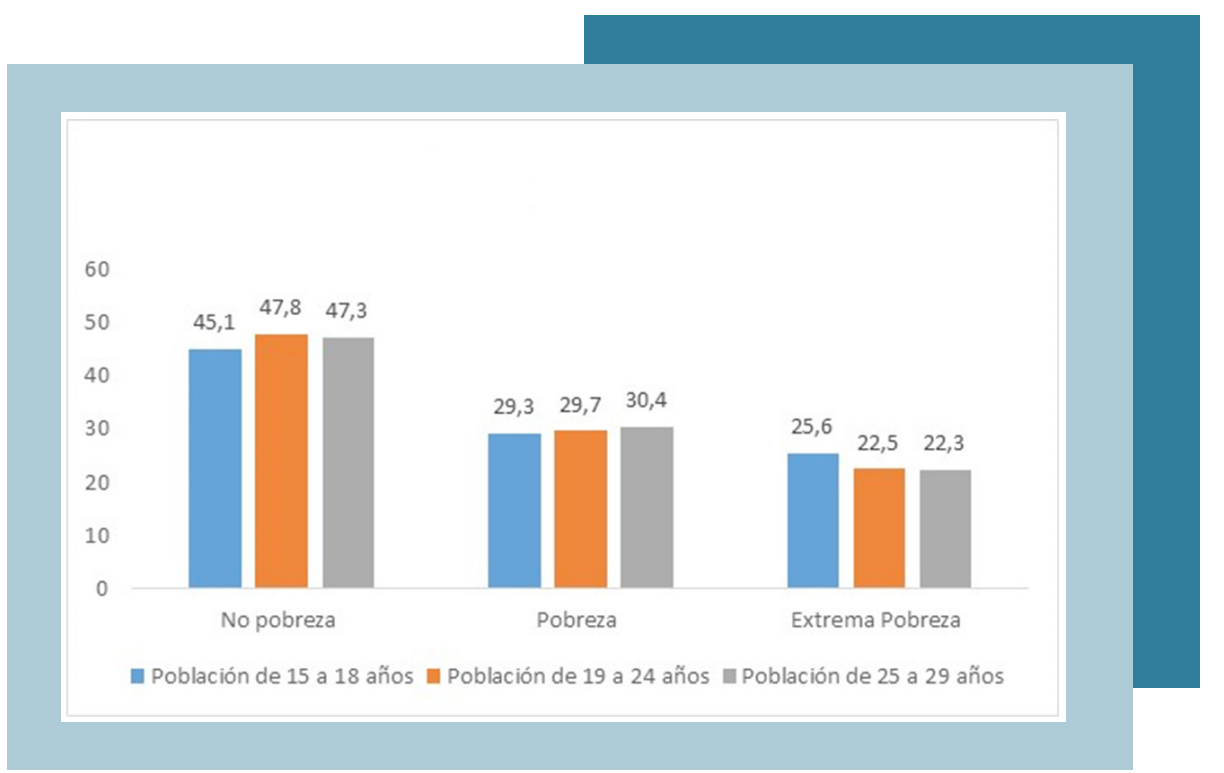

Fuente: Instituto Nacional de Estadísticas y Censos (2016)

Los GRÁfICOS 7 Y 8 muestran la variación que existe entre la población que se encuentra en condiciones de pobreza y de no pobreza por área de ubicación: en el área rural los índices se incrementan significativamente, frente a los correspondientes al área urbana (recordemos que el indicador se refiere a pobreza por NBl; esto es, servicios públicos, educación y salud) (INEC, 2016). 
GRÁFICO 7 | Jóvenes y pobreza por NBI urbano

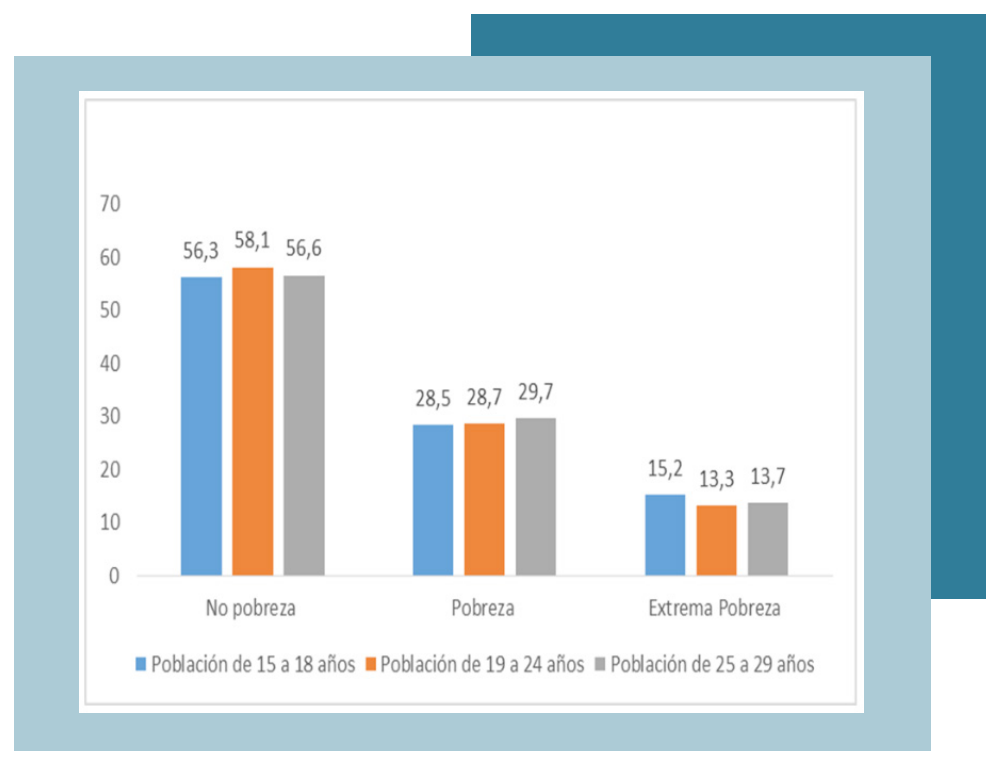

GRÁFICO 8 | Jóvenes y pobreza por NBI rural

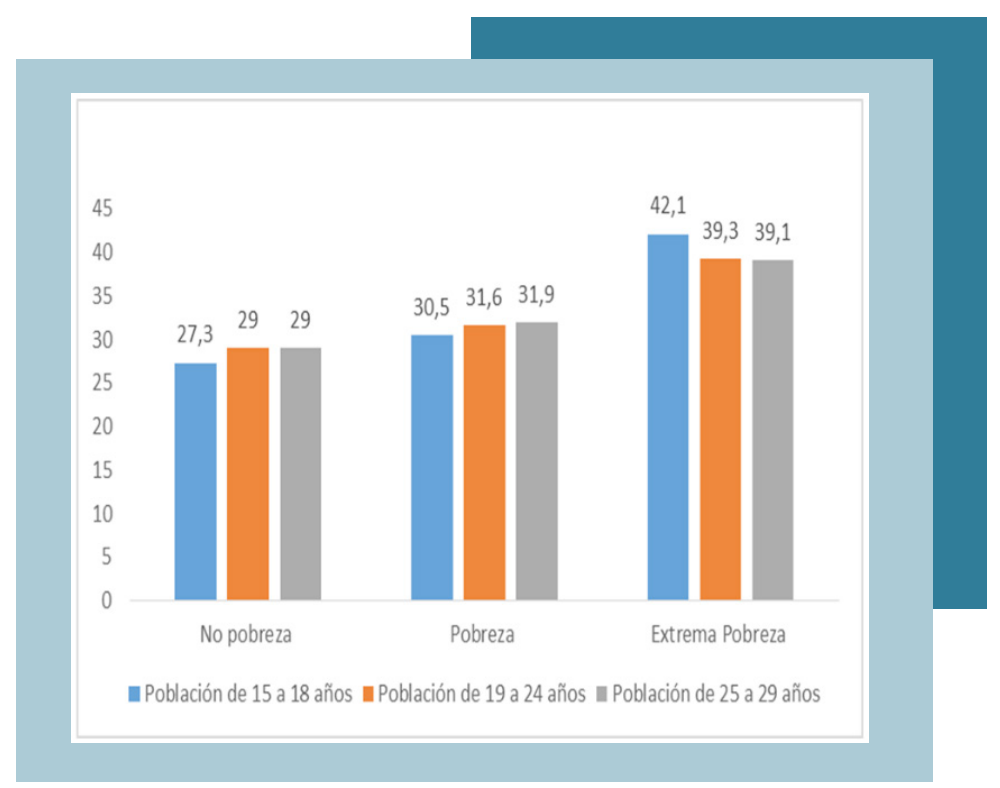

Fuente: Instituto Nacional de Estadísticas y Censos (2016) 
La tasa de participación de la población joven en la Población Económicamente Activa (PEA), como muestra el GRÁfICO 9, es del 50\%; índice que desciende desde 2005, cuando se encontraba en el 55,3\% (INEC, 2016).

GRÁFICO 9 | Tasa de participación de los jóvenes en la Población Económicamente Activa (PEA)

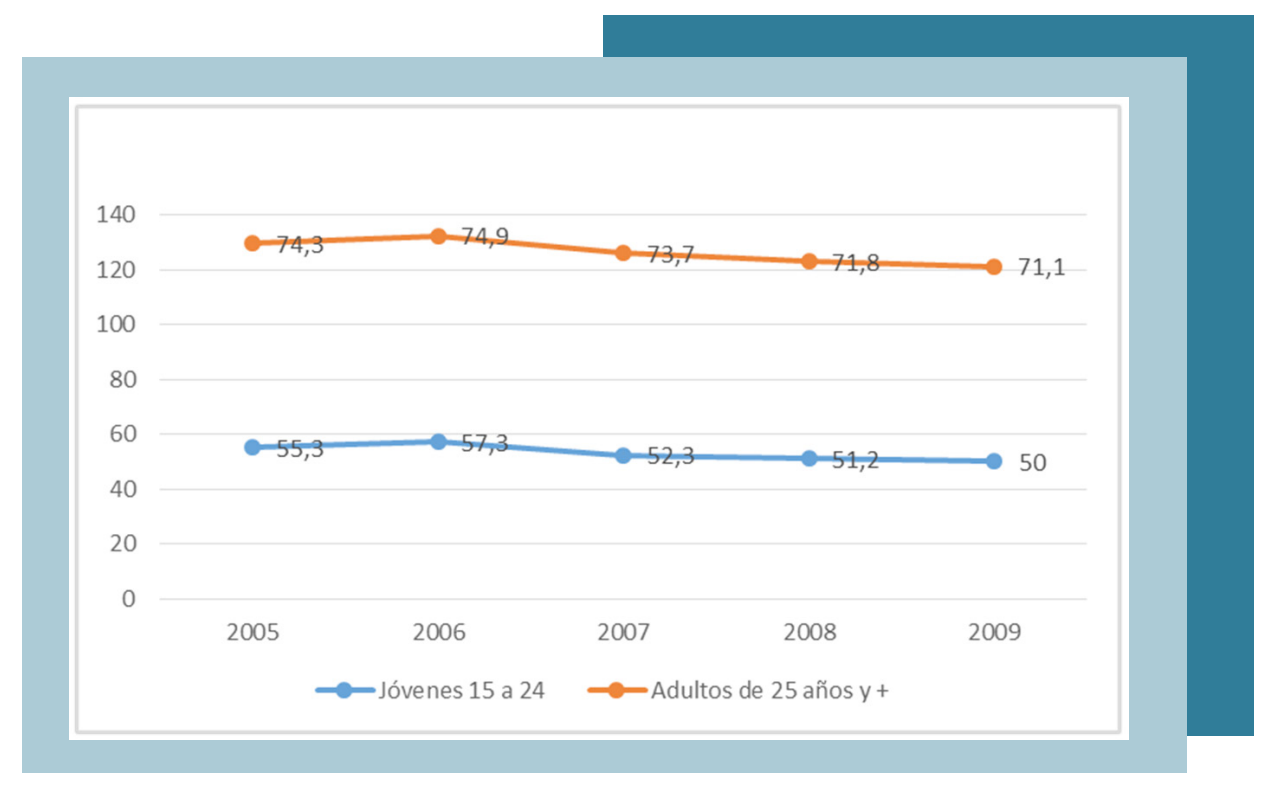

Fuente: Instituto Nacional de Estadísticas y Censos (2016)

En tanto, como muestra el GRÁFICO 10, la población joven que se encuentra ocupada en relación con la PEA representa el 42,9\% en personas de 15 a 24 años y el 67,9\% en personas mayores a 24 años (INEC, 2016). 
GRÁFICO 10 | Tasa de ocupación en la PEA

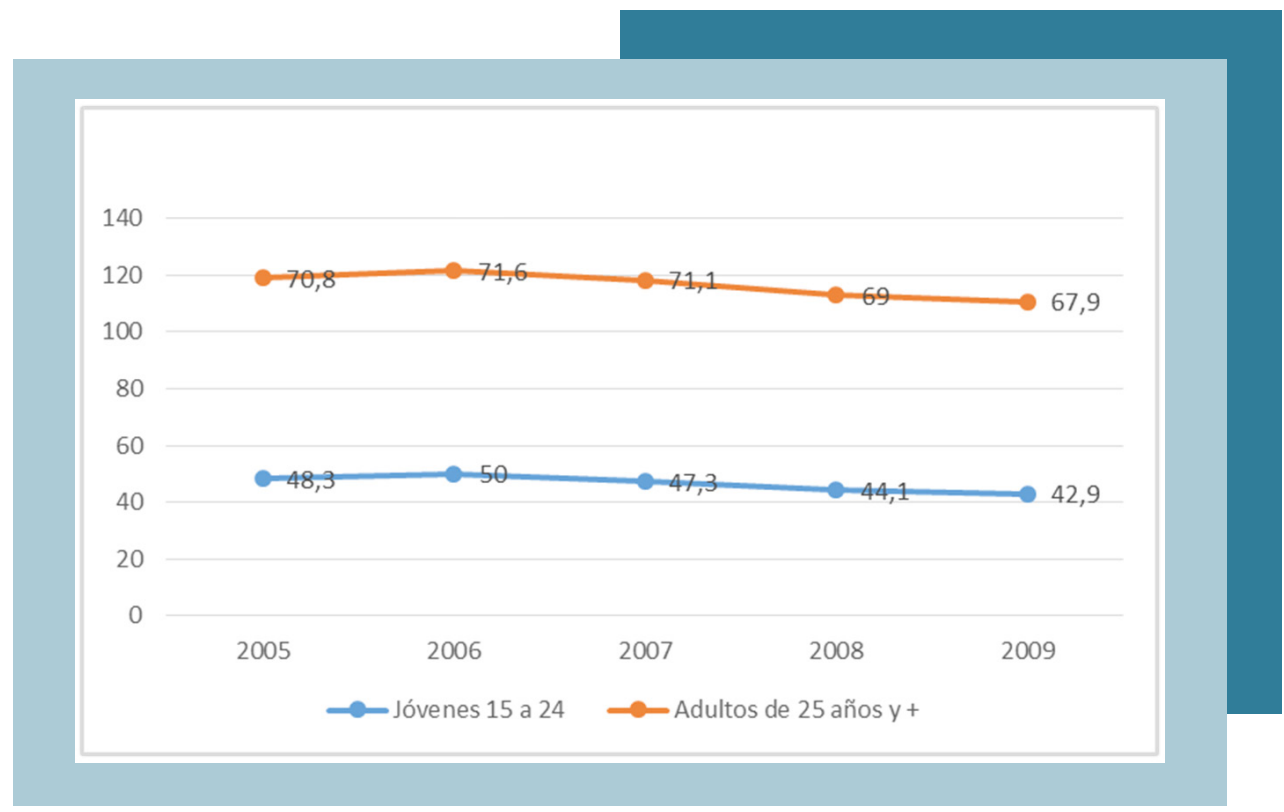

Fuente: Instituto Nacional de Estadísticas y Censos (2016)

Respecto de la tasa neta de matrículas en los distintos niveles educativos, en un comparativo entre 2001 y 2010, se observa que esta se ha incrementado. Como muestra el CUADRO 4, en el nivel primario, de un 64\% en 2001 a un 67\% en 2010; en niveles de educación básica, de un $20 \%$ en 2001 a un 28\% en 2010; en los niveles de educación secundaria, de un 37\% en 2001 a un 45\% en 2010. El nivel educativo en el que encontramos un aumento más dramático es en la educación media, que en 2001 tenía una tasa de matrícula de $7 \%$ y en 2010 de 23\%. También la educación universitaria incrementó la tasa de matriculados, de 13\% en 2001 a 22\% en 2010 (INEC, 2010). 
CUADRO 4.1 | Tasas brutas de matrícula educativa, por niveles, sexo y área de residencia

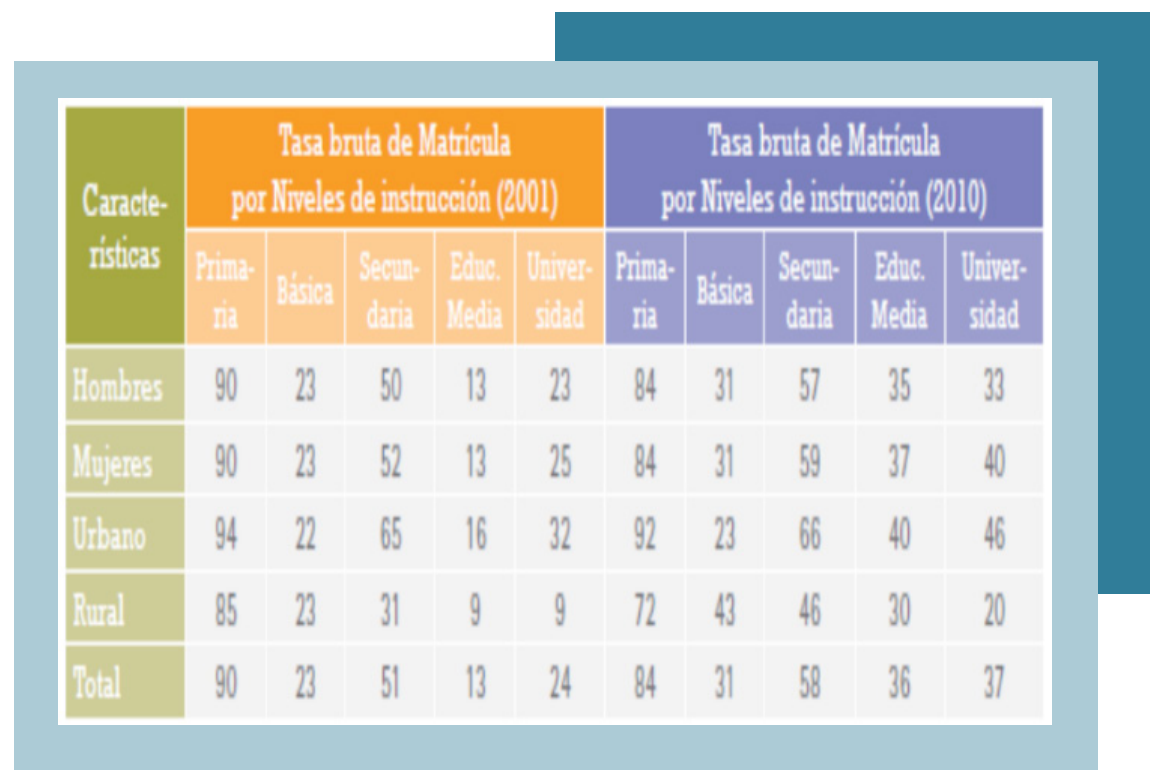

CUADRO 4.2 | Tasas netas de matrícula educativa, por niveles, sexo y área de residencia

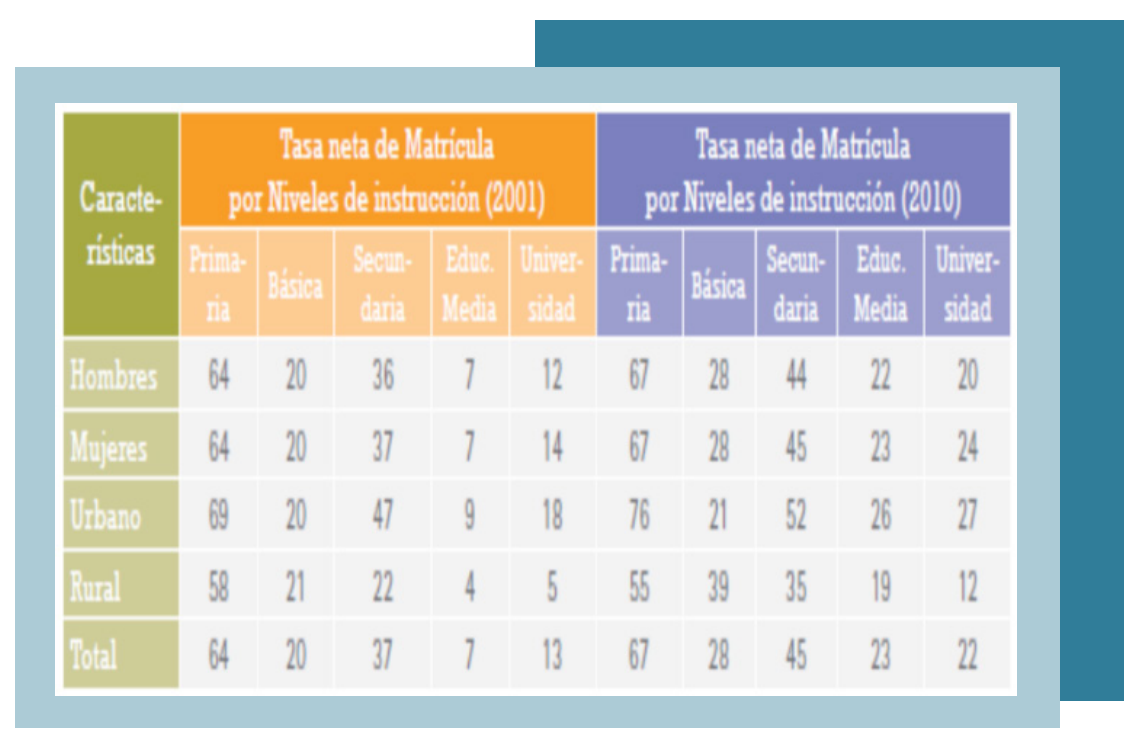

Fuente: Censo de Población 2010, INEC 
En cuanto al analfabetismo por grupo etario, el censo 2010 arrojó los siguientes datos:

CUADRO 5 | Analfabetismo por grupo etario

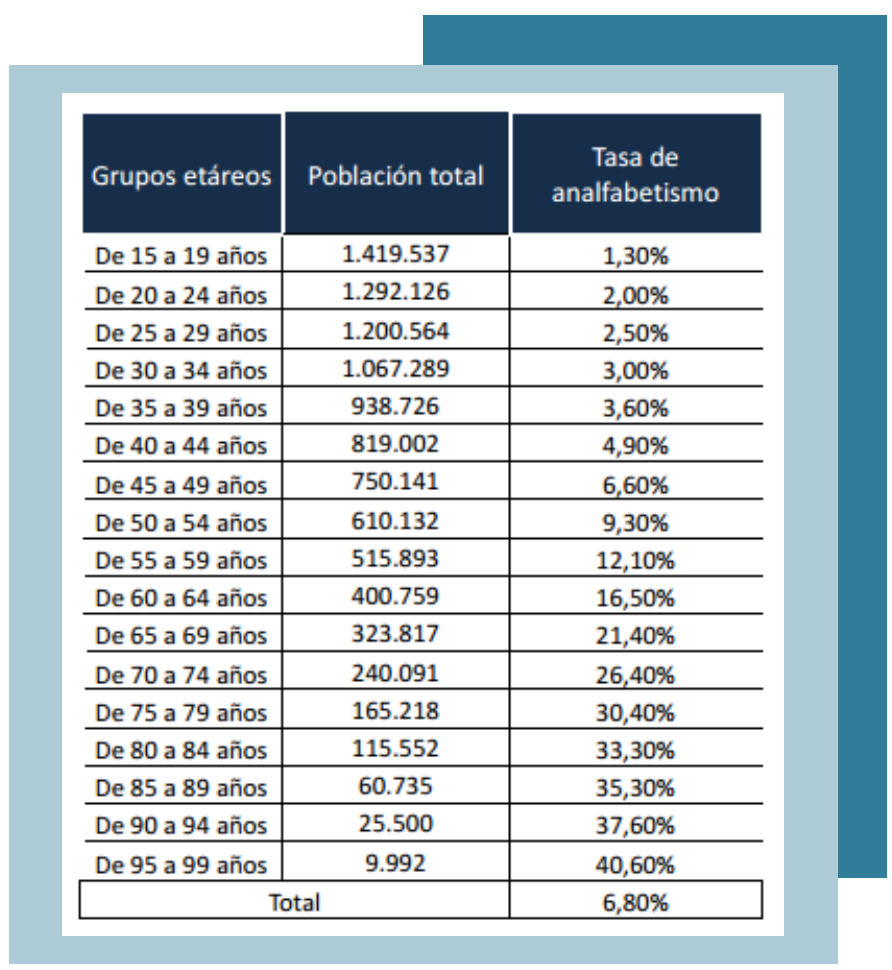

Fuente: Censo de Población 2010, INEC

\section{Implicaciones para la democracia en el Ecuador actual}

La disputa conceptual y política entre la racionalidad económica y la racionalidad política, que terminó por imponer la razón de mercado como ordenadora de un ciclo de hegemonía en la vida social del Ecuador y de los países de la región (Sánchez Parga, 2011), entró en un punto de inflexión crucial con la elección presidencial de Rafael Correa, en noviembre de 2006, y con su asunción de la conducción gubernamental del Estado, desde mayo de 2007. Desde el inicio de los sucesivos períodos gubernamentales 
de la Revolución Ciudadana, la acción pública estatal se planteó como uno de sus objetivos centrales la disminución de las enormes desigualdades existentes desde un diseño institucional caracterizado por reformas profundas en el sistema de recaudación tributaria, articuladas a la inversión pública en el sector social.

En esta dinámica, los sectores poblacionales jóvenes constituyeron uno de los principales grupos beneficiados a través de las políticas de gratuidad de la educación y la salud públicas (Restrepo \& Stefos, 2017). No obstante, y pese a los ingentes e inéditos esfuerzos realizados, la brechas de desigualdad generacional persisten, sobre todo en razón de que por primera vez en la historia demográfica del país los/as jóvenes constituyen el sector poblacional más numeroso, así como por los consabidos motivos referidos a las dificultades estructurales para su inserción al mercado de trabajo (Unda Lara, Herrera \& Llanos, 2013).

En el balance general de combate contra la pobreza los resultados son ampliamente favorables, a la luz del registro estadístico y de los principales indicadores en este rubro. Salir de la pobreza mediante el acceso a servicios públicos con estándares de calidad al alza indica que, de varios modos, ha operado un giro democrático desde la lógica de las políticas públicas en un contexto de predominancia institucional del Estado, cuestión que, siendo evaluada como necesaria en el contexto de marcada desinstitucionalización que caracterizaba al Ecuador hasta 2006, amerita una amplia y profunda revisión en la perspectiva de una mayor participación protagónica de la sociedad, teniendo como marco de referencia los principios y los objetivos inspiradores del Buen Vivir.

Se trataría, en lo fundamental, de pensar y de activar un dispositivo desde el que se genere un conjunto de condiciones que haga posible el giro de un individualismo tomado por la razón de mercado, en el que prevalece el consumidor como figura excluyente de un tejido social fragmentado y débil, hacia el reconocimiento de la individualidad en las dimensiones colectiva y social. Si, como reflexiona José Sánchez-Parga (2011), el tránsito del individualismo democrático moderno hacia el individualismo individualista se produjo en razón de una mutación cultural y política producto de la subordinación de la razón de Estado ante la razón de mercado, las estructuras de oportunidad política que se han configurado en el contexto de los gobiernos progresistas representan posibilidades ciertas de reconstituciones democráticas de individuos y de colectivos. 
En concordancia con estas ideas, la participación activa y protagónica de los y las jóvenes en el nuevo ciclo político en el que entra el Ecuador bajo el mandato presidencial de Lenín Moreno constituiría un soporte central para la activación de la memoria histórica y política de las generaciones jóvenes. Representaría el sustrato material y simbólico que sostenga y que mejore, en términos democráticos, el proyecto político que convocó a la mayoría de la sociedad ecuatoriana hace poco más de una década. 


\section{Referencias bibliográficas}

Aróstegui, J. (ed.) (2007). Generaciones y memoria. En España en la memoria de tres generaciones. De la esperanza a la reparación. Madrid, España: Complutense.

Gaitán, L. (dir.) (2010). Los niños como actores en los procesos migratorios. Implicaciones para la cooperación. Quito, Ecuador: Abya Yala.

Ortega y Gasset, J. [1923] (1966). El tema de nuestro tiempo. Madrid, España: Revista de Occidente.

Sánchez Parga, J. (2008). Valores y contravalores en la sociedad de la plusvalia. Quito, Ecuador: Universidad Politécnica Salesiana Abya Yala.

Sánchez Parga, J. (2011). «Devastación» de democracia en la sociedad de mercado. Quito, Ecuador: Centro Andino de Acción Popular (CAAP).

Unda Lara, R. y Llanos, D. (2013). Políticas públicas de juventud en Ecuador. En Alvarado, S. V., Rodríguez, E. y Vommaro, P. Políticas de inclusión social de jóvenes en América Latina y El Caribe. Situación, desafíos y recomendaciones para la acción. Buenos Aires, Argentina: CLACSO.

Unda Lara, R., Herrera, L. y Llanos, D. (2013). Estudio base para el diseño del modelo de gestión integral para la promoción del empleo en grupos de atención prioritaria y emergente, y para el diseño conceptual, operativo y de contenidos del servicio de orientación laboral para jóvenes y adolescentes. Quito, Ecuador: Centro de Investigación de la Niñez, Adolescencia y Juventud (CINAJ).

Vommaro, P. (2015). Prácticas, subjetivaciones y politizaciones: las dinámicas de movilización juvenil en la América Latina actual. En Valenzuela, J. El sistema es antinosotros. Culturas, movimientos y resistencias juveniles (pp. 429-468). México D. F., México: GEDISA / El Colegio de la Frontera Norte. 


\section{Referencias electrónicas}

Albornoz, V. (2011). Crecimiento y progreso social en el Ecuador. Quito, Ecuador: Cooperación de Estudios para el Desarrollo (CORDES). Recuperado de http://www.losexplicadores.com/vicente/articulos/15_Crecimiento_y_progreso_social.pdf

Instituto Nacional de Estadísticas y Censos (INEC) (2010). Resultados del Censo 2010 CPV: El Censo Informa: Educación. Quito, Ecuador: INEC. Recuperado de http://www.ecuadorencifras.gob.ec/wp-content/ descargas/Presentaciones/capitulo-educacion_censo_poblacion_vivienda.pdf

Instituto Nacional de Estadísticas y Censos (INEC) (2014). Informe de resultados ECV 2013-2014. Quito, Ecuador: INEC. Recuperado de https://www.unicef.org/ecuador/150411-ResultadosECV.pdf

Instituto Nacional de Estadísticas y Censos (INEC) (2016). Reporte de Pobreza por Consumo - Ecuador 2006-2014. Quito, Ecuador: INEC / Banco Mundial. Recuperado de http://www.ecuadorencifras.gob.ec/documentos/web-inec/Bibliotecas/Libros/reportePobreza.pdf

Leccardi, C., y Feixa, C. (2011). El concepto de generación en las teorías sobre la juventud. Última Década, (34), 11-32. Recuperado de www.scielo.cl/pdf/udecada/v19n34/art02.pdf

Mannheim, K. [1928] (1993). El problema de las generaciones. Revista Española de Investigaciones Sociológicas, (62), 193-242 [1993]. Recuperado de http://www.reis.cis.es/REIS/PDF/REIS_062_12.pdf

Ministerio de Inclusión Económica y Social (MIES) (2012). Jóvenes Ecuatorianos en Cifras: Indicadores clave de la década 2001-2010 y proyecciones al 2050. Quito, Ecuador: MIES. Recuperado de http://www. mdgfund.org/sites/default/files/YEM_ESTUDIO_Ecuador_Informe\%20 Situacion\%20Jovenes.pdf 
Oxfam Internacional (2017). Una economía para el 99\%. Recuperado de https://oxf.am/2t8kn2p

Restrepo, R. y Stefos, E. (2017). Atlas del Derecho a la Educación en los años de la Revolución Ciudadana: una aproximación a las transformaciones. Azogues, Ecuador: Organización de Estados Iberoamericanos (OEA) / Universidad Nacional de Educación. Recuperado de http://www. oei.es/uploads/files/news/Offices/490/atlas-derecho-educacion.pdf 